\title{
PERCEPÇÃO DE OLEOGUSTUS POR INDIVÍDUOS DE DIFERENTES ESTADOS NUTRICIONAIS ${ }^{1}$
}

\author{
PERCEPTION OF OLEOGUSTUS BY INDIVIDUALS OF \\ DIFFERENT NUTRITIONAL STATUSES
}

\section{João Paulo Lima de Oliveira ${ }^{2}$, Amanda Cristina Andrade ${ }^{3}$, Jéssica Sousa Guimarães ${ }^{4}$, Angélica Sousa Guimarães ${ }^{5}$, Sabrina Carvalho Bastos ${ }^{6}$ e Mariana Mirelle Pereira Natividade ${ }^{7}$}

\section{RESUMO}

A relação entre a aceitabilidade de alimentos e seus teores lipídicos favoreceu a descoberta de um sexto gosto básico, o oleogustus, que é despertado pelos ácidos graxos de cadeia não esterificada do tipo longa e são os responsáveis pelo verdadeiro gosto de gordura. O entendimento da percepção do oleogustus por diferentes indivíduos pode contribuir para o desenvolvimento de produtos alimentares, para a prática clínica e políticas de saúde pública. Diante disso, o objetivo deste trabalho foi avaliar a preferência sensorial pelo oleogustus por indivíduos de diferentes estados nutricionais, por meio da degustação de hambúrguer misto adicionado de diferentes concentrações de gordura, bem como identificar os fatores que influenciam o consumo de óleos e gorduras. Os hambúrgueres foram avaliados por meio de um teste de escala do ideal por 205 provadores divididos em três grupos de indivíduos: baixo peso, eutróficos e sobrepeso/obesidade. Para a análise dos dados foi utilizado o método de análise de correspondência múltipla e regressão linear simples. Os resultados demonstraram que a preferência por carnes gordurosas foi predominante no grupo de sobrepeso/obesidade, assim como o método de cocção mais utilizado por eles foi o de fritura por imersão. Este grupo também apresentou as maiores frequências de consumo diário e semanal de alimentos gordurosos ricos em gordura saturada e trans. A concentração ideal de gordura perceptível pelos grupos baixo peso, eutrófico e sobrepeso/obesidade foram de: $1,68 \%, 14,32 \%$ e $15,34 \%$, respectivamente. Dessa forma, os indivíduos com sobrepeso/obesidade possuem maior preferência por alimentos gordurosos e pelo oleogustus quando comparado com os outros grupos.

Palavras-chave: Análise sensorial, Avaliação nutricional, Obesidade, Sobrepeso, Tecnologia de Alimentos.

\section{ABSTRACT}

The relationship between the acceptability of food and their lipid contents, favored the discovery of a sixth basic taste, the oleogustus, which is awakened by long chain non-esterified fatty acids responsible for the real taste of fat. Different individuals' understanding of oleogustus perception can contribute to the development of food products, to clinical practice and public health policies. At that, the objective was to evaluate the sensory perception of oleogustus from individuals with different nutritional states, by tasting mixed hamburgers with distinct concentrations of fat in that, as well as identifying the factors that influence the consumption of oils and fats. The formulations were evaluated through a just-about-right scale by

\footnotetext{
${ }^{1}$ Trabalho de Conclusão de Curso - UFLA.

${ }^{2}$ Mestre em Nutrição e Saúde - UFLA. E-mail: joaopaulolimanut@gmail.com

${ }^{3}$ Mestre em Nutrição e Saúde - UFLA. E-mail: andradenut@gmail.com

${ }^{4}$ Doutoranda em Ciência dos Alimentos - UFLA. E-mail: jsguimaraes.nutri@gmail.com

${ }^{5}$ Doutoranda em Ciência dos Alimentos - UFLA. E-mail: aguimaraesnutri@gmail.com

${ }^{6}$ Coorientador- Docente do curso de Nutrição e do PPGNS - UFLA. E-mail: sabrina.bastos@ufla.br

${ }^{7}$ Orientadora - Docente do curso de Nutrição e do PPGNS - UFLA. E-mail: mariana.mirelle@ufla.br
} 
3 different groups: low weight, eutrophic and overweight/obesity, with a total of 205 tasters. Data was analyzed by multiple correspondence analysis and simple linear regression. The results showed preference for fatty meat was predominant in the overweight/obesity group. This group preferred cooking method was deep-frying and they had the highest frequencies of daily and weekly consumption of fatty food rich in saturated and trans fat. The ideal fat concentration perceived by the low weight, eutrophic and overweight/ obesity groups were: $1.68 \%, 14.32 \%$ and $15.34 \%$, respectively. Overweight/obese individuals have a higher preference for fatty food and by oleogustus when the results were compared with other groups.

Keywords: Sensory Analysis, Nutritional Assessment, Obesity, Overweight, Food technology.

\section{INTRODUÇÃO}

Os lipídios são uma abundante classe de substâncias encontradas nos organismos vivos, normalmente insolúveis em água, mas solúveis em solventes orgânicos (ORNELAS, 2013). Como as proteínas, ácidos nucléicos e carboidratos, os lipídios são substâncias que compõem as estruturas biológicas do organismo humano (JORGE, 2009).

Sabe-se que os lipídeos desempenham importantes funções nutricionais no organismo, fornecendo por meio da alimentação ácidos graxos essenciais (ômega 3 e ômega 6) e participando da metabolização das vitaminas lipossolúveis (ABREU; SPINELLI, 2014). Por outro lado, seu consumo excessivo é a principal causa de obesidade e de outras Doenças Crônicas Não Transmissíveis (DNCT) observadas na população contemporânea (ANS, 2017).

O elevado consumo de lipídeos se deve, em grande medida, à inserção dos alimentos ultraprocessados na dieta cotidiana. Os alimentos ultraprocessados são definidos como formulações que utilizam quantidades elevadas de substâncias extraídas dos alimentos (açúcar, sódio, óleos e gorduras) e/ou constituintes derivados de alimentos como gorduras trans e hidrogenadas (BRASIL, 2014). Sendo assim, limitar o consumo de alimentos ultraprocessados é uma forma de garantir a diminuição da ingestão de lipídios (BIELEMANN et al., 2015; ANS, 2017).

Os óleos e gorduras são uma complexa combinação de substâncias químicas, podendo ser chamados de óleos quando apresentam consistência líquida a temperatura ambiente e classificados como gorduras quando estão em forma sólida (RAMALHO; SUAREZ, 2013). A adição desses componentes em preparações culinárias pode melhorar a qualidade nutricional e sensorial quando utilizados em pequenas quantidades, aumentando a aceitação por tornarem o alimento mais palatável (FREIRE et al., 2012). Além disso, os lipídios podem melhorar a aparência, o sabor e a textura dos alimentos (DUTCOSKY, 2011), além de conferirem leveza, maciez, lubrificação, intensificarem a cor e o brilho das preparações, tornando-as mais atrativas (PHILIPPI, 2006). 
A relação estreita entre a aceitabilidade de um produto e seu teor lipídico levou à descoberta do oleogustus, termo utilizado para descrever o sexto gosto básico que o ser humano é capaz de sentir. Esse termo foi criado para descrever o gosto gerado pela ingestão de uma substância oleosa/gordurosa e não a sensação da percepção da gordura. Acredita-se que os Ácidos Graxos de Cadeia Não Esterificada (NEFA, sigla do termo em inglês Nonesterified Fatty Acids) de cadeia média e longa seriam os responsáveis por conferir o "gosto de gordura" ou oleogustus (RUNNING; MATTES, 2015).

Considerando que o consumo excessivo de lipídeos pode contribuir para o ganho de peso e o desenvolvimento de DCNT e que a ingestão destes pode ter relação com uma sensibilidade individual aumentada ao oleogustus, o entendimento da percepção desse gosto por diferentes indivíduos pode trazer contribuições para a prática clínica e políticas de saúde pública. Como os estudos sobre o oleogustus são ainda limitados e inconclusivos, são oportunas abordagens que busquem compreender a relação entre a percepção do oleogustus e o estado nutricional.

Deste modo, este estudo teve como objetivo avaliar a percepção sensorial do oleogustus em hambúrgueres mistos adicionados de gordura por indivíduos de diferentes estados nutricionais, bem como avaliar os fatores que influenciam o consumo de óleos e gorduras.

\section{MÉTODOS}

\section{ASPECTOS ÉTICOS E SELEÇÃO DOS VOLUNTÁRIOS}

O presente estudo foi aprovado pelo Comitê de Ética em Pesquisa com Seres Humanos da Universidade Federal de Lavras (UFLA) (CAAE: 61727716.7.0000.5148) e os participantes de pesquisa assinaram o Termo de Consentimento Livre e Esclarecido (TCLE). Os participantes foram recrutados aleatoriamente por meio de cartazes dispostos aos arredores do Laboratório de Análise Sensorial do Departamento de Nutrição (DNU). Foram incluídos na amostra indivíduos com idade igual ou superior a 18 anos e excluídos indivíduos que apresentassem alergia a algum dos ingredientes presentes nas preparações.

\section{PREPARAÇÃO DOS HAMBÚRGUERES}

Os hambúrgueres foram preparados no Laboratório de Técnica Dietética e Tecnologia de Alimentos (LTDTA) do DNU e a composição das cinco formulações de hambúrgueres (F1, F2, F3, F4 e F5) estão descritas na Tabela 1. A variação das formulações consistiu nas diferentes concentrações de toucinho suíno adicionado. Para se estabelecer as concentrações utilizadas no experimento foi feita uma progressão aritmética durante os pré-testes. 
Tabela 1 - Ingredientes utilizados na formulação dos hambúrgueres.

\begin{tabular}{lccccc}
\hline \multirow{2}{*}{ Ingredientes } & \multicolumn{5}{c}{ Formulações } \\
\cline { 2 - 6 } & F1 & F2 & F3 & F4 & F5 \\
\hline Peito de frango moído $(\mathrm{g})$ & 100 & 100 & 100 & 100 & 100 \\
\hline Sal $(\mathrm{g})$ & 1,5 & 1,5 & 1,5 & 1,5 & 1,5 \\
Alho $(\mathrm{g})$ & 1,0 & 1,0 & 1,0 & 1,0 & 1,0 \\
\hline Toucinho $(\mathrm{g})$ & 0 & 7 & 14 & 21 & 28 \\
\hline
\end{tabular}

Legenda: F1: formulação 1; F2: formulação 2; F3: formulação3; F4: formulação 4; F5: formulação 5.

Fonte: Construção do Autor.

As preparações foram feitas no dia anterior que precedeu a análise sensorial e foram mantidas em refrigerador Consul $1^{\circledR}$ modelo $\mathrm{CRD} 37 \mathrm{~EB}$, em temperatura entre $-5^{\circ} \mathrm{C}$ e $-15^{\circ} \mathrm{C}$. No dia da realização da análise sensorial as cinco formulações foram assadas em forno elétrico Philco ${ }^{\circledR}$, modelo PFE38P, em temperatura de $180^{\circ} \mathrm{C}$ por 30 minutos.

\section{CLASSIFICAÇÃO DO ESTADO NUTRICIONAL DOS VOLUNTÁRIOS}

Os voluntários que participaram dos testes sensoriais foram divididos em três grupos definidos de acordo com o estado nutricional, sendo: Grupo 1 (baixo peso), Grupo 2 (eutróficos) e Grupo 3 (sobrepeso/obesidades). Para a classificação do estado nutricional foi utilizado o Índice de Massa Corporal (IMC) obtido pela mensuração do peso e da altura (estatura) dos participantes. O IMC foi calculado por meio da seguinte fórmula: $\mathrm{IMC}=$ Peso corporal $(\mathrm{kg}) \div$ Estatura $\left(\mathrm{m}^{2}\right)$ e a classificação dos resultados foi realizada de acordo os pontos de corte propostos por $\mathrm{WHO}(2000)$, sendo considerado baixo peso o indivíduo com IMC $<18,5 \mathrm{Kg} / \mathrm{m}^{2}$, peso adequado entre $18,5-24,9 \mathrm{Kg} / \mathrm{m}^{2}$ e sobrepeso/ obesidade $>25,0 \mathrm{Kg} / \mathrm{m}^{2}$.

A avaliação do estado nutricional foi realizada individualmente em cada voluntário na antessala do Laboratório de Análise Sensorial, antes da realização do teste sensorial. O peso foi aferido em balança antropométrica plataforma tipo digital, com capacidade máxima de $200 \mathrm{Kg}$ e precisão de leitura de 100 g, da marca Líder ${ }^{\circledR}$, situada em piso plano sem desnível. Durante a pesagem o voluntário esteve com o mínimo de roupa possível, descalço, sem ornamentos ou objetos de metal e posicionado no centro da balança com os braços relaxados ao longo do corpo. A estatura foi aferida utilizando um estadiômetro com coluna de madeira acoplada a uma plataforma metálica, de escala até $213 \mathrm{~cm}$, precisão de $0,1 \mathrm{~cm}$, do marca Alturexata ${ }^{\circledR}$.

\section{QUESTIONÁRIO PARA INVESTIGAÇÃO DO CONSUMO DE ÓLEOS E GORDURAS}

Os voluntários foram solicitados a responderem um questionário elaborado pelos pesquisadores com o intuito de investigar o consumo de alimentos gordurosos. Dentre os itens do questionário, 
os voluntários foram questionados quanto à escolha do tipo de óleo ou gordura utilizado para cozinhar, preferência por tipos de carnes, hábito de remover gordura aparente e método de cocção preferido para o preparo dos alimentos.

Os voluntários também foram questionados quanto à frequência do consumo de alimentos ricos em gorduras (variando entre consumo diário, semanal, quinzenal, mensal, anual e não consome), sendo investigado o consumo dos seguintes itens: azeite, embutidos, queijos gordos, manteiga, margarina, maionese, calabresa e bacon. Ressalta-se que no presente estudo os dados referentes às frequências de consumo diário e semanal destacaram-se e por isso optou-se pela apresentação apenas dessas frequências.

\section{ANÁLISE SENSORIAL}

Para avaliar a percepção do gosto de óleo nos hambúrgueres foi utilizado o Teste Escala do Ideal, que é um método eficaz para indicar se a característica avaliada é extremamente perceptível ou pouquíssimo perceptível(POPPER; KROLL, 2005). O teste foi realizado pelos três grupos de voluntários descritos anteriormente, totalizando 205 provadores, dos quais 12 destes pertencem ao Grupo 1, 147 ao Grupo 2 e 46 ao Grupo 3.

As amostras de hambúrgueres foram servidas na temperatura habitual de consumo $\left(65^{\circ} \mathrm{C}\right)$, sendo que cada provador recebeu amostras contendo $20 \mathrm{~g}$ de cada formulação monadicamente, em copos descartáveis de $50 \mathrm{~mL}$ identificados com números aleatórios de três dígitos em ordem balanceada de apresentação, conforme randomização sugerida por Wakeling e Macfie(1995).

Os provadores receberam as amostras de hambúrguer e foram solicitados a avaliarem o quão ideal encontrava-se o gosto de óleo por meio de uma Escala do Ideal estruturada em 9 pontos, com a seguinte descrição: 9 - extremamente mais gorduroso que o ideal, 8 - mais gorduroso do que o ideal, 7 - moderadamente mais gorduroso que o ideal, 6 - ligeiramente mais gorduroso que o ideal, 5 - ideal, 4 - ligeiramente menos gorduroso que o ideal, 3 - moderadamente menos gorduroso que o ideal, 2 - menos gorduroso que o ideal e 1 - extremamente menos gorduroso que o ideal. Para definir a concentração ideal de gordura por grupo nas formulações de hambúrgueres, considerou-se a nota 5 como ideal e as notas superiores ou inferiores a este valor são consideradas maiores ou menores que o ideal. Os resultados da escala ideal de gordura foram discriminados por meio de análise de regressão linear simples de acordo com a classificação do estado nutricional.

\section{ANÁLISE ESTATÍSTICA}

Para as análises dos dados foi utilizado o método de análise de correspondência múltipla, que corresponde a uma análise de estatística multivariada, ou seja, é um procedimento básico de união 
de várias variáveis de modo que a análise, a descrição e a inferência são construídas por meio de respostas simultâneas de correlação entre variáveis (FERREIRA, 2011). Para a plotagem dos resultados da análise estatística multivariada foi utilizado o software The R Project for Statistical Computing (R, 2008). Os dados resultantes da Escala do Ideal foram gerados a partir da reta de regressão linear utilizando o software SensoMaker(PINHEIRO; NUNES; VIETORIS, 2013).

\section{RESULTADOS}

Dos 205 voluntários, 58,5\% pertenciam ao sexo feminino e 41,5\% ao sexo masculino, com idade entre 18 e 45 anos, predominando a faixa etária de 18 a 25 anos (76,1\%), possivelmente por se tratar de um público universitário. Em relação ao estado nutricional, 5,9\% dos voluntários possuíam baixo peso (Grupo 1), 71,7\% eram eutróficos (Grupo 2) e 22,4\% estavam acima do peso, com diagnóstico de sobrepeso ou obesidade (Grupo 3). O presente estudo buscou conhecer o perfil dos voluntários em relação ao consumo de alimentos com alto teor de gordura e compará-los com a classificação dos seus respectivos estados nutricionais. Sendo assim, na Figura 1 é apresentada a relação entre o consumo per capita de óleo e o tipo de óleo utilizado para cozinhar, em função do estado nutricional.

Figura 1 - Relação entre consumo per capita de óleo e

tipo de óleo utilizado para cozinhar em função do estado nutricional.

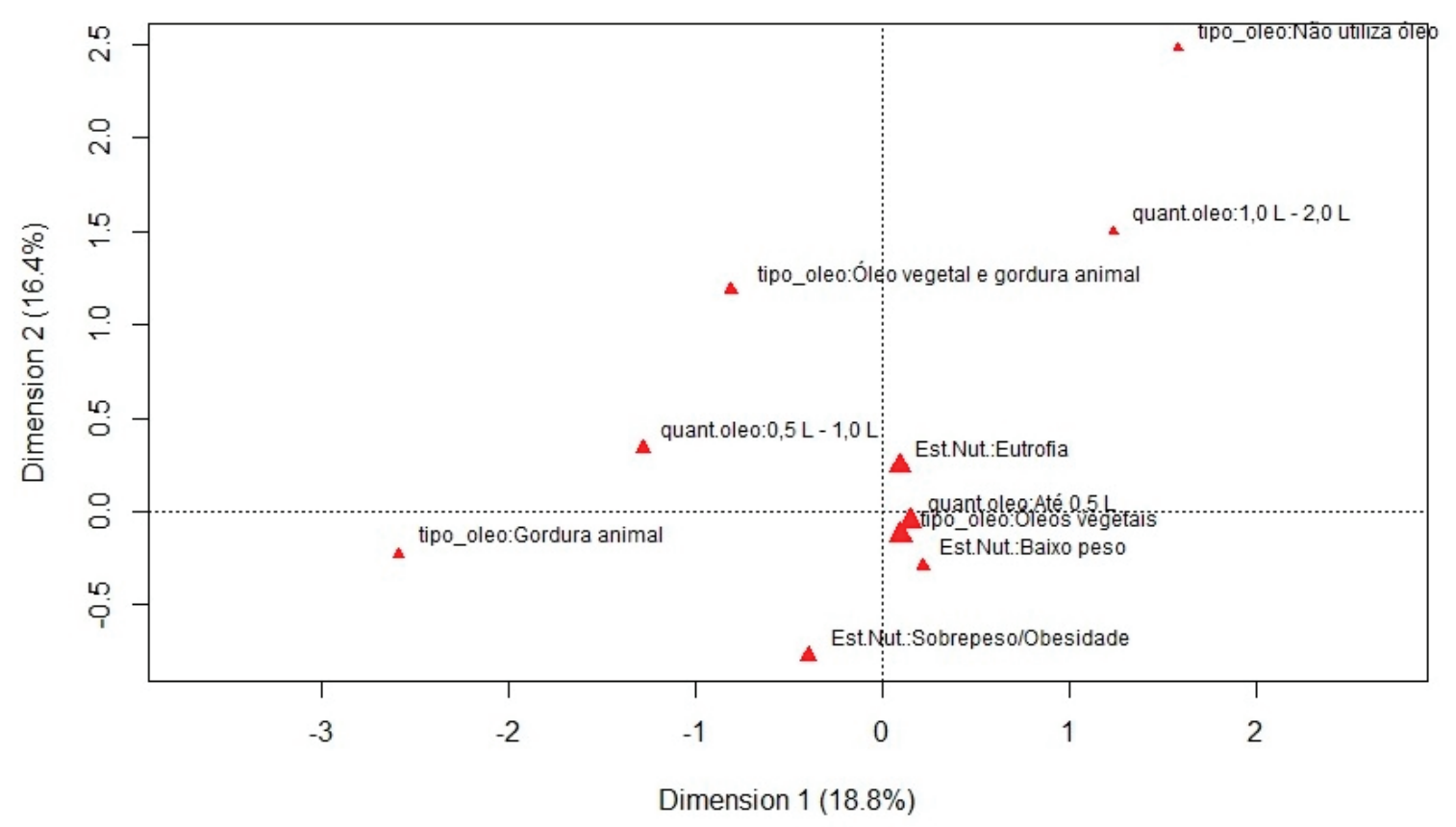

Fonte: Construção do Autor.

A Figura 2 ilustra a distribuição e a relação de proximidade entre as preferências por carnes gordurosas ou magras e o estado nutricional, além de indicar se os provadores removem a gordura da carne de acordo com a classificação do seu estado nutricional. 
Figura 2 - Relação entre o estado nutricional e o consumo de gordura em carnes

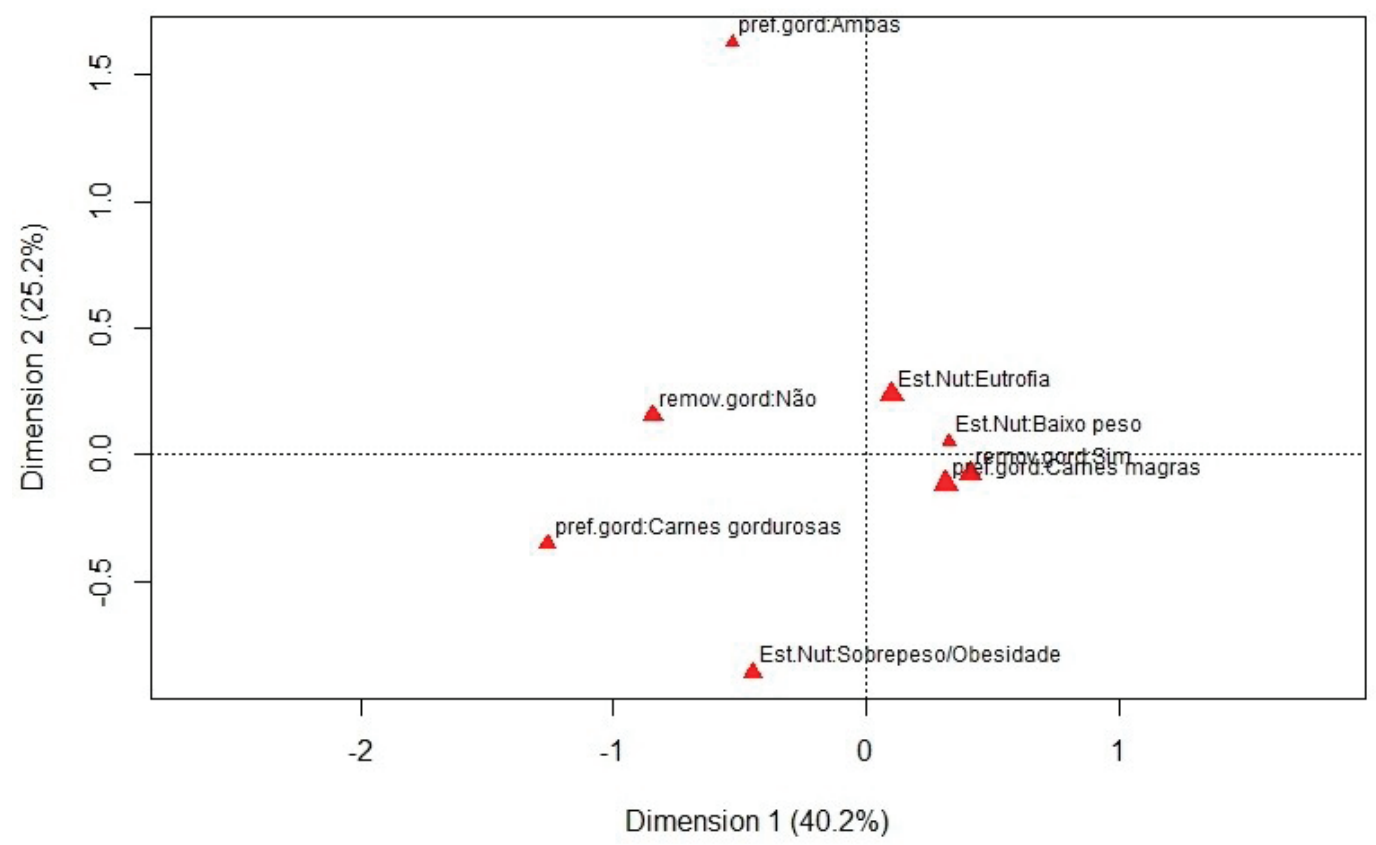

Fonte: Construção do Autor.

A Figura 3 aponta a relação entre a escolha do método de cocção e o estado nutricional dos indivíduos. Pode-se notar que os indivíduos eutróficos preferem preparações que utilizam menores quantidades de óleo, por outro lado, indivíduos com sobrepeso/obesidade preferem preparações que utilizam maiores quantidade de óleo, como por exemplo, a fritura por imersão.

Figura 3 - Escolha do método de cocção versus estado nutricional dos indivíduos

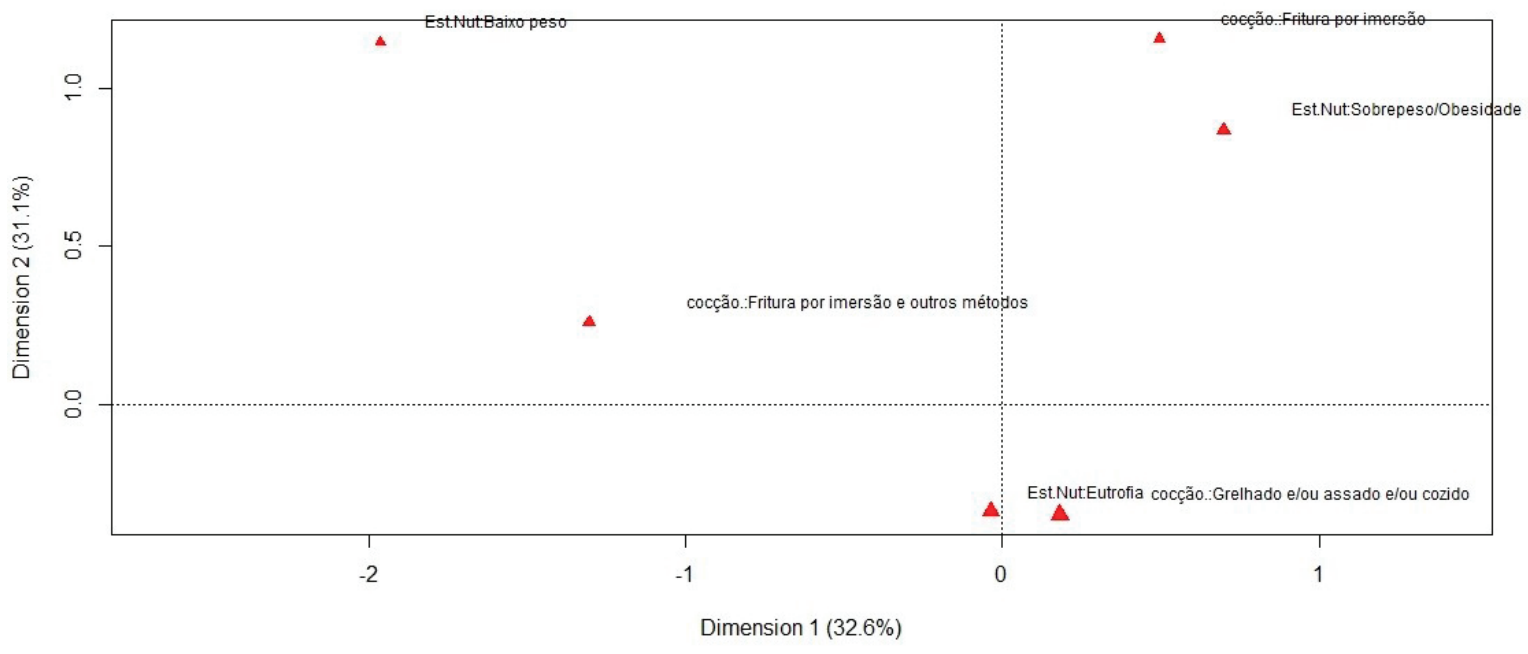

Fonte: Construção do Autor.

Pelo fato da população estudada ser universitária e por fazerem parte de uma parcela da sociedade que na maioria dos casos escolhe os alimentos devido à praticidade de preparo e consumo, foi elaborado um questionário de frequência alimentar para investigar a ingestão de alimentos gordurosos. 
Considerando que os alimentos de consumo diário e semanal são os que possivelmente mais contribuem para a qualidade da alimentação cotidiana dos indivíduos, foi avaliada a ingestão diária e semanal de alimentos gordurosos estratificada de acordo com o estado nutricional. Deste modo, na Tabela 2 são apresentados os dados de frequência de consumo diário e semanal de alimentos gordurosos de forma agrupada em função do estado nutricional. Pode-se observar que para o grupo de sobrepeseso/obesidade a soma da frequência de consumo diário e semanal de alimentos gordurosos foi mais elevada, com destaque para o consumo de queijos gordos (63\%), embutidos (58,7\%), margarina (50\%) e manteiga (47,9\%). Por outro lado, a linguiça calabresa obteve a maior frequência de consumo no grupo de baixo peso.

Tabela 2 - Frequência de consumo diário e semanal de alimentos gordurosos conforme o estado nutricional

\begin{tabular}{|c|c|c|c|c|c|c|c|c|c|}
\hline \multirow{3}{*}{ Alimentos } & \multicolumn{9}{|c|}{ Frequência de consumo por estado nutricional } \\
\hline & \multicolumn{3}{|c|}{ Baixo Peso } & \multicolumn{3}{|c|}{ Eutrofia } & \multicolumn{3}{|c|}{ Sobrepeso/Obesidade } \\
\hline & $\begin{array}{c}\text { Diário } \\
(\%)\end{array}$ & $\begin{array}{c}\text { Semanal } \\
(\%)\end{array}$ & $\begin{array}{c}\text { Total } \\
(\%)\end{array}$ & $\begin{array}{c}\text { Diário } \\
(\%)\end{array}$ & $\begin{array}{c}\text { Semanal } \\
(\%)\end{array}$ & $\begin{array}{c}\text { Total } \\
(\%)\end{array}$ & $\begin{array}{c}\text { Diário } \\
(\%)\end{array}$ & $\begin{array}{c}\text { Semanal } \\
(\%)\end{array}$ & $\begin{array}{c}\text { Total } \\
\text { (\%) }\end{array}$ \\
\hline Azeite & 33,3 & 33,3 & 66,6 & 37,4 & 26,5 & 63,9 & 42,2 & 28,9 & 71,1 \\
\hline Embutidos & 50,0 & 8,3 & 58,3 & 17,0 & 37,4 & 54,4 & 8,7 & 50,0 & 58,7 \\
\hline Queijos gordos & 0,0 & 41,7 & 41,7 & 21,2 & 39,0 & 60,2 & 23,9 & 39,1 & 63,0 \\
\hline Manteiga & 0,0 & 41,7 & 41,7 & 19,0 & 25,9 & 44,9 & 19,6 & 28,3 & 47,9 \\
\hline Margarina & 16,7 & 16,7 & 33,4 & 25,2 & 22,4 & 47,6 & 28,3 & 21,7 & 50,0 \\
\hline Maionese & 8,3 & 25,0 & 33,3 & 9,5 & 27,2 & 36,7 & 10,9 & 30,4 & 41,3 \\
\hline Calabresa & 8,3 & 33,3 & 41,6 & 1,4 & 21,1 & 22,5 & 2,2 & 23,9 & 26,1 \\
\hline Bacon & 0,0 & 25,0 & 25,0 & 0,7 & 24,5 & 25,2 & 2,2 & 32,6 & 34,8 \\
\hline
\end{tabular}

Fonte: Construção do Autor.

A Tabela 3 apresenta os resultados do ideal de concentração de gordura correspondente a cada grupo. Sendo assim, a concentração ideal de gordura para os grupos baixo peso, eutrofia, sobrepeso/ obesidade foram crescentes, com valores equivalentes a $1,68 \%, 14,32 \%$ e $15,34 \%$, respectivamente.

Tabela 3 - Concentração ideal de gordura segundo classificação do estado nutricional

\begin{tabular}{ccc}
\hline Grupos & Equação & Concentração ideal de gordura (\%) \\
\hline Baixo peso & $y=0,0649 x+4,8909\left(R^{2}=0,8423\right)$ & 1,68 \\
Eutrofia & $y=0,0706 x+3,9892\left(R^{2}=0,9408\right)$ & 14,32 \\
Sobrepeso/Obesidade & $y=0,0714 x+3,9043\left(R^{2}=0,9694\right)$ & 15,34 \\
\hline
\end{tabular}

Fonte: Construção do Autor.

\section{DISCUSSÃO}

A partir da análise da Figura 1 pode-se notar uma relação de proximidade do estado nutricional de sobrepeso/obesidade com a utilização de gordura animal, o que indica que indivíduos com este estado nutricional preparam os alimentos utilizando gordura animal. Por outro lado, observou-se que os indivíduos de baixo peso e eutróficos encontram-se próximos aos óleos vegetais, como também a quantidade per capita utilizada por esse grupo é menor (até 0,5 litro mensal). De fato, foi possível 
identificar que os indivíduos de baixo peso e eutróficos possuem o hábito de preparar os alimentos utilizando menores quantidades de óleos vegetais. A utilização de óleos vegetais tem crescido mundialmente em detrimento do consumo de gordura animal, e no Brasil, o óleo vegetal mais consumido é o óleo de soja por ser economicamente mais viável e por sua alta disponibilidade no mercado (NUNES, 2007).

Sobre a relação entre o estado nutricional e o consumo de gorduras em carnes, verificou-se que existe uma associação entre o estado nutricional e a preferência por gordura ou sensibilidade ao oleogustus, uma vez que os indivíduos eutróficos e de baixo peso têm preferência por carnes magras e se preocupam em remover a gordura aparente das carnes. Esse fato também foi notado no trabalho de Luna et al. (2014), que relatam que 82,2\% dos indivíduos diagnosticados com Hipertensão Arterial e Diabetes Mellitus possuem o hábito de remover a gordura aparente das carnes antes do consumo. E no estudo de Barbosa (2015), indivíduos com elevado grau de escolaridade e também aqueles de baixo poder aquisitivo foram os maiores consumidores de gordura saturada e trans, sugerindo que o consumo destes alimentos possa também estar relacionado a questões culturais e não apenas ao acesso à informação. É importante ressaltar que a ingestão de carne com alto teor de gordura aumenta as chances de um indivíduo desenvolver obesidade abdominal e outras doenças associadas (SOARES; BARRETO, 2014). É importante destacar que no presente estudo os indivíduos com sobrepeso/ obesidade apreciam o consumo de carnes gordurosas e preferem cozinhar utilizando gordura animal. Portanto, são indivíduos mais susceptíveis ao desenvolvimento de DCNT.

Em relação à escolha do método de cocção em função do estado, notou-se que indivíduos eutróficos têm preferência por preparações grelhadas, assadas ou cozidas, ou seja, preparam os alimentos utilizando métodos de cocção que necessitam de quantidades mínimas de óleo no preparo. Já os indivíduos com sobrepeso/obesidade preferem preparações submetidas a operações de fritura por imersão, nas quais quantidades maiores de óleo são necessárias. Estas constatações corroboram as informações descritas anteriormente, que indicaram uma apreciação do oleogustus por indivíduos com sobrepeso e obesidade.

Deste modo, percebeu-se que o método de escolha de cocção dos alimentos pode se relacionar com o estado nutricional dos indivíduos. Por outro lado, Busato et al. (2015) ressalta que a escolha pelo método de cocção dos alimentos também pode ser influenciada por costumes e hábitos adquiridos no âmbito familiar. Além disso, Freire, Mancini-Filho e Ferreira (2013) destacam que o baixo custo e a rapidez do método de fritura imersão é outro fator que amplia a utilização dessa técnica, assim como o fato da fritura atribuir características agradáveis ao alimento, como cor, sabor e textura. Considerando que alimentos fritos podem fazer parte da rotina alimentar diária de um percentual elevado de brasileiros, ações para estimular escolhas alimentares saudáveis e reduzir o consumo de alimentos ricos em gordura devem ser executadas como medidas para a redução do sobrepeso e da obesidade.

A respeito da relação entre a frequência de consumo de alimentos gordurosos e o estado nutricional, observou-se que alimentos como queijos gordos, embutidos, margarina e manteiga apresentaram 
alta frequência de consumo. Assim, destaca-se a importância de conscientizar os indivíduos para uma ingestão moderada, pois são alimentos classificados como processados e/ou ultraprocessados ricos em gordura trans e saturada, reconhecidamente prejudiciais à saúde(ANS, 2017). No presente trabalho notou-se que os indivíduos pertencentes ao grupo 3 (sobrepeso/obesidade) possuem maior preferência pelo gosto de gordura e são os maiores consumidores de alimentos gordurosos dos grupos avaliados.

Os resultados referentes à concentração ideal de gordura por meio da análise sensorial confirmam a relação entre o estado nutricional e a preferência pelo oleogustus, ressaltando que indivíduos com sobrepeso e obesidade têm preferência por alimentos com maior teor de gordura, pois fazem maior consumo per capita de óleo, utilizam gordura animal para preparar os alimentos e têm maior frequência de consumo de alimentos ricos em gordura de origem animal.

O estudo conduzido por Newman, Haryono e Keast (2013) pode contribuir para explicar os resultados encontrados no presente estudo. No trabalho citado, os autores identificaram que indivíduos com baixo peso são hipersensitivos em relação à gordura, ou seja, pouca quantidade de gordura em contato com a mucosa oral causa sensibilidade fazendo com que estes consumam menores quantidades. O contrário acontece com os indivíduos obesos, que por sua vez são hiposensíveis e por isso consomem mais gorduras por já estarem acostumados com a textura desta na mucosa oral, necessitando assim de altas quantidades para conseguirem detectá-las.

Os NEFA's são considerados os compostos mais potentes na detecção do oleogustus, sendo facilmente detectados pela cavidade oral dos seres humanos. Porém, estes ácidos graxos respondem de maneira diferente em contato com alimentos e bebidas diversificados. Além disso, as propriedades de percepção destes variam de acordo com cada indivíduo (RUNNING; MATTES, 2016).

De acordo com Tucker et al. (2014), o aumento da ingestão dietética de gordura foi associado com a diminuição da sensibilidade de detecção do gosto dos NEFA somente em indivíduos com baixo peso e sobrepeso. Contudo, a implicação nutricional ocasionada por este fato ainda carece de maiores investigações.

Em adição, Newman, Haryono e Keast (2013) relataram que a sensibilidade oral dos ácidos graxos em indivíduos obesos é uma área controversa que necessita de maiores investigações. Por outro lado, Keast (2016) defende que o limiar de detecção para o gosto de gordura está associado com a densidade energética que se ingere sugerindo que a sensibilidade ao gosto de gordura possui grandes implicações no estudo da obesidade e suas patologias associadas.

No presente estudo, os resultados apontaram que indivíduos sobrepeso/obesos possuem a maior preferência pelo gosto de gordura em comparação com voluntários eutróficos e de baixo peso.

\section{CONCLUSÃO}

No tocante à detecção do oleogustus, os indivíduos com sobrepeso/obesidade demonstraram maior preferência pelo consumo de alimentos gordurosos, confirmado pelo maior consumo per capita 
de óleo, não remoção da gordura aparente da carne, utilização da fritura como método de cocção preferencial e preferência por hambúrguer com maior teor de gordura. Estes hábitos e preferências relatados nesta pesquisa sugerem uma relação entre o estado nutricional e a preferência por alimentos oleosos/gorduras, notando-se que indivíduos com sobrepeso e obesidade apreciam o oleogustus.

\section{REFERÊNCIAS}

ABREU, E. S.; SPINELLI, M. G. N. Seleção e preparo de alimentos: gastronomia e nutrição. São Paulo: Metha, 2014.

AGÊNCIA NACIONAL DE SAÚDE SUPLEMENTAR (ANS) - BRASIL. Manual de diretrizes para o enfretamento da obesidade na saúde suplementar brasileira. Rio de Janeiro: ANS, 2017.

BARBOSA, L. P. Consumo de gorduras e escolaridade em adultos: estudo de base populacional no município de Campinas, SP. 2015. 68 p. Dissertação (Mestrado em Saúde Coletiva) - Faculdade de Ciências Médicas, Universidade Estadual de Campinas, Campinas, 2015.

BIELEMANN, R. M. et al. Consumo de alimentos ultraprocessados e impacto na dieta de adultos jovens. Revista de Saúde Pública, São Paulo, v. 49, n. 28, p. 1-10, maio 2015.

BRASIL. Ministério da Saúde. Secretaria de Atenção à Saúde. Departamento de Atenção Básica. Guia Alimentar para a População Brasileira. 2. ed. Brasília: Ministério da Saúde, 2014.

BUSATO, M. A. et al. Ambiente e alimentação saudável: percepções e práticas de estudantes universitários. Semina: Ciências Biológicas e da Saúde, Londrina, v. 36, n. 2, p. 75-84, jul./dez. 2015.

DUTCOSKY, S. D. Análise sensorial de alimentos. 3. ed. Curitiba: Champagnat, 2011.

FERREIRA, D. F. Estatística multivariada. 2. ed. Lavras: Editora UFLA, 2011.

FREIRE, A. C. A. et al. Aspectos nutricionais e sensoriais da redução do teor de gordura em preparações de carne bovina. Revista da Universidade Vale do Rio Verde, Três Corações, v. 10, n. 2, p. 270-278, ago./dez. 2012. 
FREIRE, P. C. M.; MANCINI-FILHO, J.; FERREIRA, T. A. P. C. Principais alterações físico-químicas em óleos e gorduras submetidos ao processo de fritura por imersão: regulamentação e efeitos na saúde. Revista de Nutrição, Campinas, v. 26, n. 3, p. 353-358, maio/jun. 2013.

JORGE, N. Química e tecnologia de óleos vegetais. São Paulo: Cultura Acadêmica, 2009.

KEAST, R. S. J. Effects of sugar and fat consumption on sweet and fat taste. Current Opinion in Behavioral Sciences, v. 9, n. 1, p. 55-60, June 2016.

LUNA, J. L. et al. Consumo de frutas, vegetais, óleos e gorduras e nível de atividade física em população portadora de hipertensão arterial e/ou diabetes mellitus adscrita à Estratégia de Saúde da Família em Salvador, Bahia. Revista Brasileira de Pesquisa em Saúde, Vitória, v. 16, n. 3, p. 122-130, jul./set. 2014.

NEWMAN, L.; HARYONO, R.; KEAST, R. Functionality of fatty acid chemoreception: a potential factor in the development of obesity? Nutrients, v. 5, n. 4, p. 1287-1300, Apr. 2013.

NUNES, S. P. Produção e consumo de óleos vegetais no Brasil. Boletim Eletrônico do Departamento de Estudos Socioeconômicos Rurais, Paraná, n. 159, p. 1-10, jun. 2007.

ORNELAS, L. H. Técnica e dietética: seleção e preparo dos alimentos. 8. ed. São Paulo: Atheneu, 2013.

PHILIPPI, S. T. Nutrição e técnica dietética. 2. ed. Barueri: Manole, 2006.

PINHEIRO, A. C. M.; NUNES, C. A.; VIETORIS, V. SensoMaker: a tool for sensorial characterization of food products. Ciência e Agrotecnologia, Lavras, v. 37, n. 3, p. 199-201, maio/jun. 2013.

POPPER, R; KROLL, D. R. Just-about-right scales in consumer researchers. ChemoSense, v. 7, n. 3, p. 3-6, June 2005.

R DEVELOPMENT CORE TEAM. 2009. R: A language and environment for statistical computing. R Foundation for Statistical Computing, Vienna, Austria. URL: https://www.R-project.org/.

RAMALHO, H. F.; SUAREZ, P. A. Z. A química dos óleos e gorduras e seus processos de extração e refino. Revista Virtual de Química, Niterói, v. 5, n.1, p. 2-15, jan./fev. 2013. 
ROSADO, E. L.; MONTEIRO, J. B. R. Obesidade e a substituição de macronutrientes da dieta.

Revista de Nutrição, Campinas, v. 14, n. 2, p. 145-152, ago. 2001.

RUNNING, C. A.; CRAIG, B. A.; MATTES, R. D. Oleogustus: the unique taste of fat. Chemical Senses, Oxford, v. 40, n. 7, p. 507-516, Sept. 2015.

RUNNING, C. A.; MATTES, R. D. A review of the evidence supporting the taste of non-esterified fatty acids in humans. Journal of the American Oil Chemists's Society, v. 93, n.10, p. 1325-1336, Oct. 2016.

SOARES, D. A.; BARRETO, S. M. Sobrepeso e obesidade abdominal em adultos quilombolas, Bahia, Brasil. Cadernos de Saúde Pública, Rio de Janeiro, v. 30, n. 2, p. 341-354, fev. 2014.

TUCKER, R. M. et al. Associations between BMI and fat taste sensitivity in humans. Chemical Senses, v. 39, n. 4, p. 349-357, May 2014.

WAKELING, I. N.; MACFIE, J. H. Designing consumer trials balanced for first and higher orders of carry-over effect when only a subset of $k$ samples from $t$ may be tested. Food Quality and Preference, v. 6, n. 4, p. 299-308, 1995.

WORLD HEALTH ORGANIZATION. Obesity: preventing and managing the global epidemic. Report of a World Health Organization Consultation. Geneva: WHO Obesity Technical Report Series, v. 894 , p. $253,2000$. 
